\title{
BROWNIAN MOVEMENTS IN GASES AT LOW PRESSURES.
}

By R. A. Millikan.

THE essential improvements which the "oil drop method"1 introduced into the study of Brownian movements consisted in

I. Enabling the observer to hold a given particle under observation as long as desired, even in a rarified gas, by counteracting the downward pull of gravity by the upward pull of an electrical field.

2. Eliminating completely all uncertainty as to the resistance offered by the medium to the motion of the drop through it.

This last result was accomplished by combining the Brownian movement equation

$$
\bar{D}=\sqrt{\frac{4}{\pi} \frac{R T}{N K} t}
$$

in which $R$ is the gas constant, $T$ absolute temperature, $N$ the number of molecules in a gram molecule, and $K$ the unknown resistance factor, with the characteristic equation of the "oil drop method," viz.,

$$
e=\frac{K}{F}\left(v_{1}+v_{2}\right)_{1},
$$

in which $F$ is the electrical field strength, $e$ the elementary electrical charge and $\left(v+v_{2}\right)_{1}$ the greatest common divisor of the series of values assumed by the sum of speeds $\left(v_{1}+v_{2}\right)$ as the drop changes charge through the capture of ions. The resulting equation which gives the average Brownian displacement is

$$
\bar{D}=\sqrt{\frac{4}{\pi} \frac{R T\left(v_{1}+v_{2}\right)_{1} t}{F(N e)}},
$$

1 R. A. Millikan, Phys. Rev., XXXII., p. 349, April, I9II.

2 This is merely the Einstein equation $\Delta x^{2}=2 R T / N K \cdot t$ modified so as to give the average displacement instead of the average of the squared displacements by substituting from the Maxwell distribution law wihch holds for Brownian displacements as well as for molecular velocities $\bar{D}=\overline{\Delta x}=\sqrt{2 / \pi} \sqrt{\overline{\Delta x^{2}}}$.

${ }^{3}$ This equation is merely equation I, p. 353 (PHYs. REv., XXXII.), viz. $e_{n}=\frac{m g}{F v_{1}}\left(v_{1}+v_{2}\right)$ after $\frac{m g}{v_{1}}$ has been replaced by $K$ (which is constant for a given drop) and after the whole equation has been divided through by $n$, the number of elementary charges in $e_{n}$. 
an equation which differs from Brownian movement equations which preceded it in being completely independent of all assumptions as to the density or shape of the Brownian particle, or as to the law of motion of the particle through the medium. It contains nothing but accurately measurable elements and is actually found to yield under all circumstances calculated values of $\bar{D}$ which agree with observed values within the limits of experimental error (about 4 per cent. for the individual drops worked with, though only 0.6 per cent. for the mean of all the drops used). ${ }^{1}$

Another forward step in the study of Brownian movements becomes possible as soon as we become able to determine the constant $K$. This constant is defined by the equation

$$
X=K v_{1},
$$

in which $X$ is the force acting on the particle and $v_{1}$ the velocity produced by this force. This step has now been taken by working with oil drops at all pressures from $5 \mathrm{~mm}$. up by the methods already referred to $(l . c$., I, p. I). The details of this work are shortly to be published in full, but the result which gives the law of motion of a drop of radius $a$ through a gas of viscosity $\mu$, and mean free path $l^{1}$ under the influence of a force $X$ is contained in the empirical equation

$$
X=6 \pi \mu a v_{1}\left\{\mathrm{I}+\frac{l}{a}\left(.874+.32 \epsilon^{-1.5} \frac{a}{l}\right)\right\}^{-1}
$$

$\epsilon$ representing the Naperian base. This means that $K$ of equations I, 2, and 4 , is given by

$$
K=6 \pi \mu a\left\{\mathrm{I}+\frac{l}{a}\left(.874+.32 \epsilon^{-1.54 \frac{a}{l}}\right)\right\}^{-1} .
$$

This makes it possible to determine precisely how the Brownian displacements $\bar{D}$ increase as the pressure decreases in the case of a drop of any given radius. A glance at equation ( $\mathbf{I}$ ) shows, since $\mu$ is independent of pressure, that

$$
\bar{D} \propto \sqrt{\mathrm{I}+\frac{l}{a}\left(.874+.32 \epsilon^{-1.54 \frac{a}{l}}\right)} .
$$

The accompanying table shows how $\bar{D}$ increases as the pressure falls from $760 \mathrm{~mm}$. to I mm. in the case of the smallest drops $(a=.00003 \mathrm{~cm}$.) upon which accurate experiments have thus far been made in the use of the "oil drop method."

1 Harvey Fletcher, Phys. Rev., XXXIII., August, I9II. R. A. Millikan, Popular Science Monthly, 80, p. 438, May, I9I2; also Trans. Am. Electrochemical Soc., Vol. XXI., p. I85, April, rgr2. 


\begin{tabular}{c|c|c|c}
\hline \hline$p \mathrm{~mm}$. Hg. & $l \mid a$ & $A^{\prime}$ & $\left.\bar{D} / \bar{D}_{1}\right)$ \\
\cline { 2 - 4 } 760 & .314 & .874 & 1.13 \\
380 & .628 & .902 & 1.25 \\
190 & 1.255 & .968 & 1.49 \\
100 & 2.385 & 1.042 & 1.88 \\
50 & 4.770 & 1.106 & 2.50 \\
25 & 9.540 & 1.142 & 3.45 \\
10 & 23.85 & 1.174 & 5.39 \\
5 & 47.70 & 1.184 & 7.58 \\
2 & 119.25 & 1.190 & 11.95 \\
1 & 238.5 & 1.192 & 16.89 \\
\hline \hline
\end{tabular}

The column headed $A^{\prime}$ in the table gives the variable values which must be assigned to the quantity $A$ if the law of motion of a drop through a resisting medium is to be expressed in the form

$$
X=6 \pi \mu a v_{1}\left(\mathrm{I}+A^{\prime} \frac{l}{a}\right)^{-1} .
$$

$A^{\prime}$ is constant and has the value .874 so long as $l / a<.4$, after which it rises as shown on the table to the value I.193, beyond which it never goes (see also equation 3 ). The column headed $\bar{D} / \bar{D}_{0}$ in the table gives the ratio between the Brownian displacement at the given pressure, and the displacement at a pressure so high that the term in $l / a$ is negligible in comparison with unity. The values given in this last column are computed by means of ( 7 ) but all save the last three of them have been checked experimentally with no larger errors than those mentioned above.

The table brings out clearly the advance which the "oil drop method" introduced into the study of Brownian movements by making it possible to hold a given particle under observation for an indefinite time in a gas at any pressure from $\mathrm{I} \mathrm{mm}$. up. For example, the ratio between the Brownian displacement $\bar{D}_{a}$ of a given particle in air at atmospheric pressure where the term in $l / a$ is nearly negligible and the Brownian displacement $\bar{D}_{w}$ of the same particle in water is

$$
\frac{\bar{D}_{a}}{\bar{D}_{w}}=\sqrt{\frac{\mu_{u}}{\mu_{a}}}=\sqrt{\frac{.0110}{.00178}}=7.7 .
$$

The displacement of the particle considered above is multiplied again 7.6 fold by reducing the pressure to $5 \mathrm{~mm}$. of mercury (see table). The change from $760 \mathrm{~mm}$. to $5 \mathrm{~mm}$. corresponds then very closely to the transfer of the particle from water to air.

${ }^{1} l$ is computed from $\mu=.3502 m n \bar{c} l$, in which $\bar{c}$ is the average molecular velocity and $\mu_{t}=.0001824+.00017856\left(t-23^{\circ}\right.$ C. $) .00276$. 
I have not yet carried the direct quantitative measurements to pressures lower than $5 \mathrm{~mm}$., but I expect to do so in the near future and see no difficulties in the way of working at pressures as low as I $\mathrm{mm}$. Under these conditions the Brownian displacements of the particle considered will be more than Ioo times greater than they are in water, and the Brownian movement method of determining the elementary electrical charge should begin to be comparable in accuracy with the oil drop method, although its probable error would still be five or six times as great as that inherent in the latter method. The present value of $e$ by this last method is

$$
e=4.774 \pm .009 \text {. }
$$

The value by the Brownian movement method fluctuates between Perrin's value $4.2 \times$ IO $^{-10}$ and Fletcher's value $5.01 \times 10^{-10}$ with Svedberg's determination at the intermediate value $4.7 \times$ I0 $^{-10}$.

De Broglie, ${ }^{1}$ who has made qualitative observation on the Brownian movement of smoke particles at low pressures, concluded that $\bar{D}$ was independent of pressure down to about $\mathrm{I} \mathrm{cm}$. of mercury, where it began to increase rapidly. This would be roughly true for particles ten times the size of those above considered, $i$. e., for particles of radius $a-.000 \underline{3}$ $\mathrm{cm}$. for the value of $l / a$ at $\mathrm{I} \mathrm{cm}$. would then be 2.385 (see table) and $\bar{D}$ would be but $1.87 / \mathrm{I} .13=\mathrm{I} .66$ times as much as at atmospheric pressure.

RYERSON LABORATORY,

The University of Chicago,

December 20, I9r2.

1 De Broglie, C. R., I54, p. II2, January, I9I2. 